\title{
Psychological correction of mild cognitive impairments in the elderly
}

\author{
Dmitry Savchenko ${ }^{1, *}$, Natalya Belyakova ${ }^{1}$ \\ ${ }^{1}$ Russian state social University, Moscow, Russia
}

\begin{abstract}
The article discusses in detail the features of cognitive functions in the elderly with mild cognitive impairment. The aim of this research was to develop, implement and study the impact of psycho-correctional programs on mild cognitive impairment in the elderly. This article focuses on such cognitive functions as memory, attention, and thinking. We used the following methods to implement the research goal and objectives: theoretical analysis of scientific literature on the research topic; psychodiagnostic methods: Wechsler memory scale (WMS), the "10 words of Luria" method, Schulte tables, Munsterberg's test, methodology Simple Analogies, and Object Exclusion methods. Wilcoxon Signed-Rank Test for matched pairs, a method of mathematical statistics, was used to process the data obtained. Based on the initial diagnosis, a program was developed for psychological correction of cognitive functions in the elderly with mild cognitive impairment, which included a number of therapies of a certain duration. Based on the repeated diagnostics, recommendations were developed to use the psychological correctional program by psychologists, clinical psychologists working with the elderly, as well as for the people of this age to maintain their cognitive functions within the individual norm and their relatives.
\end{abstract}

\section{Introduction}

Today, a human life expectancy has increased, owing to a general significant increase in the world standard of living. According to data provided by Rosstat, the average life expectancy in Russia has increased to 73.6 years from the beginning of 2019. First of all, this is due to successful public health policy and socio-economic development.

At the same time, with the increase in life expectancy, the society as a whole and specialists in gerontology in particular face new complex challenges related to the elderly adaptation, their health strengthening and opportunities expanding. According to WHO, share of the world's population aged over 60 will double by 2050 from about $11 \%$ to $22 \%$. According to V. Hachinski the absolute number of people aged 60 years and older will increase over the same period to 2 million people [1].

Cognitive functions in the elderly undergo some changes. According to P.S. Sachdev, D.M. Lipnicki, Z.S. Khachaturian, L.H. Kuller, D. Ding, Q. Zhao, if there are no serious diseases leading to dementia, such as Alzheimer's disease, cerebral atherosclerosis and

*Corresponding author:d1990s@mail.ru 
organic brain disorders, then most of the mental abilities in old age are practically unchanged, but the processing speed of cognitive information decreases [2, 3, 4]. By the age of 60 , there may be a slight decrease in memory, forgetfulness, distraction, fatigue, "confusion of thoughts", difficulties in understanding and mastering new material, and difficulties in the ability to work with several sources of information simultaneously.

Often we are facing the delusion that the impaired memory and other cognitive functions are the rule in older age.

According to J.A. Elman, A.J. Jak, A.J. Petkus, S.M. Resnick, a tolerant attitude ultimately leads to the development of mild disorders in moderate, and after a few years, people may experience the first serious signs of dementia [5, 6]. Timely diagnosis of cognitive functions and preventive measures significantly reduce the risk of mild cognitive impairment and prevent the development of moderate cognitive impairment and dementia, and allow you to keep them at the level of individual norm. In addition, in recent years, there has been increased interest in this matter both in this country and abroad. Various government programs appear aimed at maintaining the life and health of elderly people, such as the "Moscow Longevity" project by S.S. Sobyanin, Mayor of Moscow. It also contributes to their greater satisfaction with life.

At the present stage, such scientists as E. Dove, A.J. Astell, J. Budgett, A. Brown, F. Lam, M. Huang $[7,8,9]$ have conducted research on the mild cognitive impairment. However, it is worth noting that these experts examined mild cognitive impairment in the elderly using medical approach, while we apply some psychological methods in our work.

The classification of the severity of cognitive impairment, according to A. Serrano-Pozo, J.H. Growdon, understands mild cognitive impairment as a decrease in cognitive abilities compar ed to a higher premorbid level of an individual that formally remains within the average statistical norm or slightly deviates from it [10]. Most often, a patient himself expresses this in his complaints, but it is not so noticeable to the others, causes no difficulties in everyday life, even in its most complex forms. According to A. Ortega, JC. Stevens, who reported at the National Academy of Neuropsychology in 2008, from a neuropsychological point of view, any age-related changes in the brain, firstly, affect the neurodynamic factors of cognitive functions associated with the structures of the first functional (energy) block [11]. These changes lead to a slowdown of mnestic processes. Aging of an individual also depends on many other factors. According to S. Teipel, M.J. Grothe, the functional potential of the human biological system begins to grow from the first years of life and reaches its peak at an early young age, and then naturally decreases [12]. In turn, the decline rate of the biological system potential is determined by our behavior and impact of many other factors throughout the life; for example, from what a person eats, how physically active he is, and to what extent he has been exposed to risk factors associated with smoking, harmful alcohol addiction or exposure to toxic substances throughout the life.

According to R. Ihl, M. Tribanek, metabolic and nootropic drugs (Cerebrolysin, Noopept, Piracetam, etc.) are used to treat mild cognitive impairment, and vitamin therapy is also used [13]. The number of older people continues to grow from year to year; therefore, according to C. Di Lorito, P. Andrés, H. Vico, so that they feel comfortable in modern society, in order to keep up with the pace of its development, they must maintain their cognitive functions at the level of an individual norm, that is, not only take medicine, but also constantly perform exercises aimed at mnemonic functions, mental activity and concentration training $[14,15]$. Therefore, aim of this research was to develop, implement and study the impact of psychological correction programs on mild cognitive impairment in the elderly. 


\section{Materials and methods}

To study mild cognitive impairment in the elderly, we used the following methods: theoretical analysis of scientific literature on the research topic; psychodiagnostic methods: Wechsler memory scale (WMS) (D. Wexler), the "10 Words of Luria" method (A.R. Luria), Schulte Tables (V. Schulte), Munsterberg's test (G. Münsterberg), the methodology "Simple Analogies" (modified by I.Yu. Kulagina and V.N. Kalyutsky), the "Exclusion of Objects" method (S.Ya. Rubinstein). Wilcoxon Signed-Rank Test for matched pairs, a method of mathematical statistics, was used to process the data obtained.

An empirical study was organized and implemented in the Medical Rehabilitation Unit (MRU) of the Clinic of Memory based on the branch of Psychoneurological Dispensary No. 9 of the State Budgetary Institution P. B. Gannushkina Psychiatric Clinical Hospital No. 4 (Moscow). The study involved 24 elderly individuals, including 21 women and 3 men, with mild cognitive impairment (memory, attention, and thinking) whose average age was $70.5 \pm$ 3.1 years.

The Medical and Rehabilitation Unit of the Clinic of Memory for the elderly accommodates 50 patients, divided into 6 groups. We separated the patients according to the following principle:

- Groups 1 and 2: patients with preserved cognitive functions, the most "strong" groups;

- Groups 3 and 4: patients with mild cognitive impairment;

- Groups 5 and 6: patients with the first signs of dementia, moderate cognitive impairment; Sessions in the Clinic of Memory MRU were held in small groups of 8-10 people. Based on these terms, we accounted patients from groups 3-4 with mild cognitive impairment, as well as 4 people from group 2, whose cognitive indicators were close to the selection boundaries in groups 3-4 according to the results of diagnosis by clinical psychologists of the Clinic.

When choosing the test subjects, we proceeded from the following parameters:

1. Subjects were within the age range from 61 to 75 years (older subjects were accounted according to WHO classification);

2. Patients of the Clinic of Memory MRU who attended sessions in groups 2-4;

3. Absence of diagnosed diseases (psychiatrists of the Medical and Rehabilitation Unit of the Clinic of Memory made diagnosis "for the elderly") such as:

- Alzheimer's disease;

- Dementia (cortical, subcortical, multifocal and cortical-subcortical);

- Dyscirculatory encephalopathy;

- Vascular cerebral disorder, etc.

\section{Results}

Primary diagnosis of cognitive functions.

Having selected a test battery, we diagnosed cognitive functions (memory, attention, and thinking) of the elderly attending sessions at the Clinic of Memory MRU.

\subsection{Memory Diagnosis}

\subsubsection{Wexler's Memory Scale method}

The target of this method is to examine various types of memory and it consists of seven (7) subtests. According to the initial diagnosis results, we noted that according to Wexler Memory Scale, the majority of test subjects (70.8\%) showed a significant decline in logical 
memory (subtest 4). The remaining seven (7) people (29.2\%) had an average value of this indicator. In addition, some subjects experienced inconsistency during retelling, "jumping" from the beginning to the end of the story, which demonstrated the mnestic impairment.

In addition, we noticed the reduced results for subtest 6 "Fixative memory" (4.2\%) and subtest 7 "Associative memory" (8.3\%). Average results for subtest 6 were observed in $66.7 \%$ of subjects, and high - in $29.2 \%$. Average results for subtest 7 were observed in 19 people $(79.2 \%)$ and high - in 3 people $(12.5 \%)$. These results attest to the subjects' ageappropriate ability to retain information about current events, as well as to establish logical connections between concepts.

All 24 subjects $(100 \%)$ showed a high level of subtests 1 "Orientation and awareness" and 2 "Orientation in time and space". This means that the elderly individuals are aware of where they are here and now, have a good knowledge of social issues, as well as knowledge of basic data about themselves.

In subtest 3 "Mental control", $87 \%$ of the subjects have a high level of mental control and $13 \%$ have an average level, which indicates a good voluntary control over their actions in most elderly people.

In subtest $5,83 \%$ of the subjects showed average results, while $17 \%$ had high results. These data indicate that mechanical memory in the elderly is within the average range.

We also calculated the Absolute Index of Memory (AIM) and the Equivalent (to the intelligence) Index of Memory (EIM). The absolute index of memory is calculated by adding up scores for all 7 subtests, receiving the so-called "raw scores". The equivalent index of memory is calculated by adding an age correction to AIM, calculated for persons in the range from 15 to 79 years. Then, the age-corrected result is converted into estimates that correspond to the scores of the standard IQ indicator.

Therefore, when calculating the received data, we noted that AIM in all elderly people (24 people, 100\%) was within the average value. While EIM in 15 people $(62.5 \%)$ was at a high level, in 5 people $(20.8 \%)$ - at an average level and in 4 people $(16.7 \%)$ - at a low level. This distribution of values allows us to state that the intellectual abilities of most elderly people with MCI are not declined.

Thus, we conclude that logical, fixation, and associative memory in the elderly are the main types of memory that need correction.

\subsubsection{The "10 Words of Luria» method}

According to the 10 Words of Luria method, the obtained results of long-term memory affect all levels of manifestation. For example, in half of the subjects (12 people, 50\%), the longterm memory is within the average norm. We noticed that a quarter of the subjects ( 6 people, $25 \%$ ) had high results and a quarter had low results (2 people, $8.3 \%$ ), and 4 people $(16.7 \%)$ showed the results below average.

Thus, we can conclude that the elderly people with MCI have difficulties in maintaining information in long-term memory, since the number of people with a high level for this indicator is only a quarter of the total number of subjects.

\subsection{Attention Diagnosis}

\subsubsection{Schulte Tables method}

This method is used to evaluate such indicators as work efficiency (WE), working capacity (WC), and psychological stability (PS). Work efficiency indicators divided almost evenly between the average level that $54 \%$ of the subjects have scored and the low level that $46 \%$ 
of the subjects have scored, and demonstrates that the elderly need more time for a task, which means they have difficulty in concentrating on any activity.

The degree of working capacity in the elderly also turned out to be in the range of average (20 people, $83 \%$ ) and low (4 people, 17\%) values. These indicators show us that the elderly need a lot of time to understand the principle of the task and get involved in work.

The indicator of psychological stability, like the previous ones, is in the range of average (11 people, 54\%) and low (13 people, 46\%) values. Such results indicate that it is difficult for the elderly to concentrate on any activity for a long time.

Thus, using Schulte Tables method, we have noticed that all analyzed indicators are at the average and low levels, which supposes that such attention characteristics as concentration and stability need correction.

\subsubsection{Munsterberg's test}

The test aimed at studying the selectivity of attention gave the following results. 15 subjects $(62 \%)$ showed an average level for this indicator, 7 subjects $(30 \%)$ showed a high level, and 2 subjects $(8 \%)$ showed a low level. Analyzing the obtained data, we concluded that the elderly had a sufficiently developed ability to select significant incentives and ignore secondary ones.

\subsection{Thinking Diagnosis}

\subsubsection{Methodology "Simple Analogies"}

This methodology aims at studying the features of logical thinking. The calculation revealed approximately the same number of people with high and average level of logical thinking: $10(41.7 \%)$ and $11(45.8 \%)$ people, respectively, and only $3(12.5 \%)$ subjects have a level below the average.

The implementation of this method requires the test subject to understand the logical connections and relationships between concepts, and each new comparison bases on a new principle. Since the number of errors was insignificant, and no low results were observed, we were able to assume that the elderly with MCI continue to be able to operate with abstract concepts through reasoning.

\subsection{2 "Exclusion of Objects" method}

Finally, we evaluated the method, aimed at examining such mental operations as comparison, abstraction and generalization, analysis and synthesis, according to the number of correct answers. Having analyzed the results of this method, we found that 19 people $(79.2 \%)$, i.e. the majority of test subjects, had an average level of mental operations, and 5 people $(20.8 \%)$ had a high level.

Thus, since the calculation did not reveal any low results, we can conclude that the elderly with MCI have no difficulty establishing similarities and differences between concepts and identifying their common features.

Development of a psychological correction program.

Based on the theoretical review data and results of initial measurement of empirical research, we developed a corrective program, the purpose of which was the psychological correction of long-term memory, logical, associative, fixative and mechanical memory, stability and concentration of attention, ability to summarize, logical thinking, analysis processes and synthesis. 
The corrective program includes 8 sessions held 2 times a week for 20-25 minutes in groups of 8-10 people. This program added the work carried out by psychologists of the Clinic of Memory MRU. Each contained exercises aimed at working with memory, attention and thinking in a complex and at separate sessions aimed at individual training of each cognitive function.

\section{Discussion}

Repeated diagnosis of cognitive functions. Having implemented a full program of psychological correction of cognitive functions in the elderly with mild cognitive impairment, we made a repeated diagnosis in order to determine the effectiveness of the elaborated program.

\subsection{Memory Research}

\subsubsection{Wechsler Memory Scale Method (D. Wexler)}

Having implemented the psycho-correctional program, we noted positive changes in the indicators of the following subtests. Thus, Logical Memory subtest 4 and Associative Memory subtest 7 are of statistical significance, where $\mathrm{T}_{\mathrm{emp}} .=54.5$ and $\mathrm{T}_{\mathrm{emp}} .=43$, respectively, with $\mathrm{p} \leq 0.01$. Therefore, we can conclude that older people have become better at remembering material based on logical associations. These results may also indicate an improvement in short-term aural memory, since tasks from these subtests were read aloud, and the subject's task was to retain and reproduce this information.

Fixation Memory subtest 6 showed an improvement in fixation memory following the corrective program, which was confirmed by the statistical significance of $\mathrm{T}_{\mathrm{emp}} .=41.5$ at $\mathrm{p} \leq 0.05$. The results obtained indicate an improvement in short-term visual memory, since the subject's task was to reproduce the drawing from memory, after 10 seconds of presentation.

We also discovered statistically significant differences in terms of absolute index of memory (AIM), which is calculated by adding up scores of all 7 subtests, and equivalent (to the intelligence) index of memory (EIM), which is calculated by adding an age correction to AIM. The results, where $\mathrm{T}_{\text {emp. }}=44$ and $\mathrm{T}_{\text {emp. }}=47$, respectively, at $\mathrm{p} \leq 0.01$ indicate that, in general, the overall memory index in the elderly with mild cognitive impairment increased after the psycho-correctional program, and their intellectual abilities were not declined.

Thus, according to the results of Wexler Memory Scale method, we can conclude that the psycho-correctional program has a positive effect on logical, associative and fixative types of memory, which the improvement of the overall memory indicator confirms.

\subsubsection{The "10 Words of Luria" method (A.R. Luria)}

Using this method, we examined the long-term memory in the elderly with MCI. Having analyzed the results through the mathematical statistics method, Wilcoxon T-test, we found the statistical significance of this indicator $\left(\mathrm{T}_{\mathrm{emp}}=14.5\right.$, with $\left.\mathrm{p} \leq 0.01\right)$. This suggests that the corrective program had a positive impact on the ability for long-term storage of information in the memory of the elderly.

\subsection{Attention Research}




\subsubsection{Schulte Tables (V. Schulte)}

Using this method, we examined such indicators of attention as working capacity (WC) and psychological stability (PS), as well as calculated the work efficiency (WE). The results of statistical analysis of this method showed that work efficiency (WE) significantly improved $\left(\mathrm{T}_{\text {emp. }}=5.5\right.$, at $\mathrm{p} \leq 0.01$ ), but at the same time indicators of working capacity (WC) and psychological stability (PS) did not have any statistically significant differences.

Thus, we can conclude that the corrective program does not have a significant impact on the time required to be included in the work (IW) and on the ability to concentrate on any activity for a long time (PA). At the same time, it helps to reduce the time that older people spend on the method implementation in general (WE).

\subsubsection{Munsterberg's test. (G. Munsterberg)}

The results of statistical analysis did not reveal any significant changes in the indicator of selectivity of attention after the corrective program. It is worth noting, that when completing the assignment in the second measurement, the test subjects became more focused and the evidence of selectivity was at "medium" and "high" levels.

However, despite the fact that there were no statistically significant differences in the selectivity of attention, a tendency to improve this indicator was noticed in the group of the elderly who completed the program of psychological correction.

\subsection{Thinking Research}

\subsubsection{Methodology "Simple Analogies" (modified by I.Yu. Kulagina and V.N. Kalyutsky)}

Since the objective of this methodology is to study characteristics of thinking, namely, understanding logical connections and relationships between concepts, the analysis was carried out on the number of correct answers. Having analyzed the results through the method of mathematical statistics, the Wilcoxon T-test, we found that an increase in the number of correct answers to the tasks after completing the corrective program had a statistical significance within the range $\mathrm{p} \leq 0.05$, where $\mathrm{T}_{\mathrm{emp}} .=28$.

Thus, we can state an improvement in the process of logical thinking, since the number of errors on performing the task has decreased.

\subsection{2 "Exclusion of Objects" method (S. Ya. Rubinstein)}

The objective of this method is to examine such mental operations as analysis and synthesis, abstraction and generalization; therefore, we made the analysis of the results on the number of correct answers. According to the results of statistical processing, we can conclude that the corrective work has reduced the number of errors, which is confirmed by statistical significance, where $T_{\text {emp. }}=12.5$, with $\mathrm{p} \leq 0.01$. This indicates that the elderly with mild cognitive impairment, when analyzing the card, began to choose the answers closest to the logical rules more often. In other words, the choice of a "generic" concept, which is closest to three of the four objects depicted, became more logical, and did not base on the individual preferences of the test subject.

Thus, such thinking processes as the ability to generalize, logical thinking, analysis and synthesis in the elderly with MCI improved after completion of the psycho-correctional program. 


\section{Conclusions}

The performed empirical research demonstrated that the program of psychological correction of mild cognitive impairment in the elderly had a positive effect on long-term, logical, associative and fixative memory, and logical thinking, as well as on such mental operations as analysis and synthesis, abstraction and generalization. There were no any statistically significant improvements in changes in concentration and selectivity indicators.

Thus, we can conclude that the psychological correction of mild cognitive impairment has a positive effect on memory processes and mental operations, while at the same time it turned out to be much more difficult to identify an improvement in concentration and selectivity indicators.

Based on the obtained data, we have developed recommendations that can be useful for psychologists, clinical psychologists working with the elderly, as well as people of this age category and their relatives to maintain their cognitive functions within the individual norm. Since psychologists and clinical psychologists work with MCI elderly people mainly in medical institutions, the principal recommendations for them, when implementing a corrective program in a group, are limited to maintaining a positive psycho-emotional atmosphere, as well as monitoring the correctness of tasks. One of the most important recommendations for relatives of the elderly with mild cognitive impairment is to monitor the daily performance of tasks of the psycho-correctional program. Every corrective program has an instruction for each exercise, so it will not cause any difficulties to understand the task. Recommendations for using psychological correction program for the elderly focus on making them understand why they need this cognitive training, and directly on their doing one or two exercises daily.

\section{References}

1. V. Hachinski, Einhäupl K., Ganten D., Alladi S., et al., Alzheimer's and Dementia 15(7), 961-984 (2019) https://doi.org/10.1016/j.jalz.2019.06.001

2. P.S. Sachdev, D.M. Lipnicki, J. Crawford, et al., PLoS ONE 8(3), e59649 (2013) https://doi.org/10.1371/journal.pone.0059649

3. Z.S. Khachaturian, L.H. Kuller, A.S. Khachaturian, Alzheimer's and Dementia 15(7), 865-869 (2019) https://doi.org/10.1016/j.jalz.2019.06.003

4. D. Ding, Q. Zhao, Q. Guo, X. Liang, J. Luo, L. Yu, L. Zheng, Z. Hong, Alzheimer's \& Dementia: Diagnosis, Assessment \& Disease Monitoring 4, 28-36 (2016) doi:10.1016/j.dadm.2016.03.004

5. J.A. Elman, A.J. Jak, M.S. Panizzon, et al., Alzheimer's \& Dementia: Diagnosis, Assessment \& Disease Monitoring 10, 372-381 (2018) doi:10.1016/j.dadm.2018.04.003

6. A.J. Petkus, S.M. Resnick, S.R. Rapp, et al., Alzheimer's \& Dementia: Translational Research \& Clinical Interventions 5, 118-128 (2019) doi:10.1016/j.trci.2019.02.003

7. E. Dove, A.J. Astell, Alzheimer's \& Dementia: Translational Research \& Clinical Interventions 5, 475-482 (2019) doi:10.1016/j.trci.2019.07.008

8. J. Budgett, A. Brown, S. Daley, T.E. Page, S. Banerjee, G. Livingston, A. Sommerlad, Alzheimer's \& Dementia: Diagnosis, Assessment \& Disease Monitoring 11, 45-52 (2019) https://doi.org/10.1016/j.dadm.2018.11.001

9. F. Lam, M. Huang, L. Liao, R. Chung, T. Kwok, M. Pang, Journal of Physiotherapy 64(1), 4-15 (2018) ISSN 1836-9553, https://doi.org/10.1016/j.jphys.2017.12.001

10. A. Serrano-Pozo, J.H. Growdon, Journal of Alzheimer's Disease 67, 795-819 (2019) doi: $10.3233 / \mathrm{JAD} 181028$ 
11. National Academy of Neuropsychology, Archives of Clinical Neuropsychology 23(6), 627-751 (2008) https://doi.org/10.1016/j.acn.2008.08.001

12. S. Teipel, M.J. Grothe, M. Dyrba, J. Zhou, J. Sepulcre, C. Sorg, C. Babiloni, Journal of the International Neuropsychological Society 22(2), 138-163 (2016) doi: $10.1017 / \mathrm{S} 1355617715000995$

13. R. Ihl, M. Tribanek, N. Bachinskaya, Pharmacopsychiatry 45(2), 41-46 (2012) doi http://dx.doi.org/ 10.1055/s-0031-1291217

14. C. Di Lorito et al., Maturitas 122, 8-21 $\quad$ (2019) https://doi.org/10.1016/j.maturitas.2019.01.001

15. P. Andrés, H. Vico, A. Yáñez, A. Siquier, G.A. Ferrer, Alzheimer's \& Dementia: Diagnosis, Assessment \& Disease Monitoring 11, 108-114 (2019) doi:10.1016/j.dadm.2018.12.002 